\title{
Analysis of the Expression and Prognosis for Leukocyte Immunoglobulin-Like Receptor Subfamily B in Human Liver Cancer
}

\section{Jing Fan}

The second hosptial of Nanjing,Nanjing university of Chinese medicine

\section{Lili Wang}

The second hosptial of Nanjing, Nanjing university of Chinese medicine

\section{Miao Chen}

Nanjing university of Chinese medicine

\section{Jiakang Zhang}

Nanjing university of Chinese medicine

\section{Jiayan Li}

The second hospital of Nanjing,Nanjing university of Chinese medicine

\section{Fangnan Song}

The second hospital of Nanjing,Nanjing university of Chinese medicine

\section{Aidong Gu}

The second hospital of Nanjing,Nanjing university of Chinese medicine

\section{Dandan Yin}

The second hospital of Nanjing, Nanjing unviersity of Chinese medicine

Yongxiang Yi ( $\square$ ian0126@126.com )

The second hospital of Nanjing,Nanjing university of Chinese medicine https://orcid.org/0000-00029465-1212

\section{Research Article}

Keywords: liver cancer, LILRB, prognosis, bioinformatics analysis, NK cells

Posted Date: November 2nd, 2021

DOI: https://doi.org/10.21203/rs.3.rs-752285/v2

License: (c) (1) This work is licensed under a Creative Commons Attribution 4.0 International License.

Read Full License 
Version of Record: A version of this preprint was published at World Journal of Surgical Oncology on March 24th, 2022. See the published version at https://doi.org/10.1186/s12957-022-02562-w. 


\section{Abstract}

Background: Leukocyte immunoglobulin-like receptor subfamily B (LILRB), including 5 subtypes, is a group of inhibitory receptors in immune system. The LILRB family is known to be involved in the tumor progression of various cancer types, especially liver cancer. However, the expression patterns and prognostic values of LILRB family members in liver cancer tissues remain unclear.

Methods: We used the Oncomine database, GEPIA database, Kaplan-Meier Plotter, Timer, TISIDB and cBioPortal to assess the expression and prognostic value of the LILRB family in liver cancer patients. We also verified the expression of the LILRB family in tumor tissues and tumor-free liver tissues at the protein level by using immunohistochemistry. The STRING website was used to explore the interaction between the LILRB family and their related genes. The DAVID database was used to perform the GO and KEGG analyses. Flow cytometry was used to assess the infiltrated NK cells in liver cancer tissues.

Results: Our study revealed that the mRNA expression of LILRB1, LILRB2, LILRB3 and LILRB5 was downregulated, while compared with normal tissues, the mRNA expression of LILRB4 was upregulated in liver cancer tissues. Survival analysis revealed that LILRB2 and LILRB5 mRNA expression levels were significantly associated with OS and DSS and that the mRNA expression of all LILRB family members was significantly correlated with RFS and PFS. Next, we further found that the mRNA expression of all LILRB family members was significantly associated with the infiltration of $\mathrm{B}$ cells, $\mathrm{CD} 8^{+} \mathrm{T}$ cells, $\mathrm{CD} 4^{+} \mathrm{T}$ cells, macrophages, and neutrophil dendritic cells in liver cancer. Finally, GO and KEGG analyses found that the LILRB family and its related genes were involved in antigen processing and presentation and natural killer cell-mediated cytotoxicity pathways.

Conclusions: Our study suggested that LILRB family expression was associated with the prognosis of liver cancer patients and infiltrated immune cells. The LILRB family might be involved in antigen processing and presentation and natural killer cell-mediated cytotoxicity pathways.

\section{Background}

Primary liver cancer is the sixth most diagnosed cancer and the fourth deadliest cancer in the world ${ }^{[1]}$. Asia and Africa have the highest incidence rates of liver cancer ${ }^{[2 ; 3]}$. In China, liver cancer is the fourth most commonly diagnosed cancer ${ }^{[4]}$. In addition, there are more male patients than female patients. According to pathological types, primary liver cancer is divided into hepatocellular carcinoma (HCC),

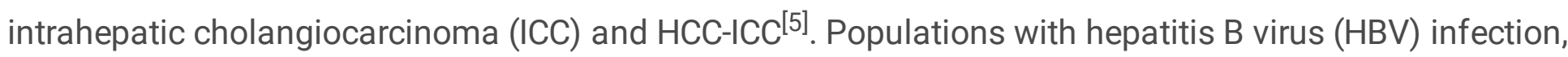
hepatitis $\mathrm{C}$ virus (HCV) infection, nonalcoholic fatty hepatitis and alcohol abuse have a high risk of liver cancers. Classic clinical screening methods are alpha-fetoprotein (AFP) and liver ultrasonography. Nuclear medical imaging and liver puncture biopsy are used to estimate the prognosis of liver cancer patients. Considering the heterogeneity of liver cancer, the current method to screen or to predict the prognosis of liver cancer has limitations. Therefore, the identi 1 cation of alternative and novel biomarkers is urgently needed for successful liver cancer treatment. 
The leukocyte immunoglobulin-like receptor B (LILRB) family is a group of immune inhibitory receptors. The LILRB family consists of five members: LILRB1, LILRB2, LILRB3, LILRB4, and LILRB5. In an earlier study, the LILRB family was found to be expressed on many immune cells, such as dendritic cells, macrophages, $B$ cells, T cells and NK cells ${ }^{[6]}$. Our previous study also discovered that LILRB2 in CD1c ${ }^{+}$ myeloid DC subsets was remarkably increased in PBMCs of HCC patients and the microenvironment of liver cancer ${ }^{[7]}$. Engagement of LILRB1 and LILRB2 with its ligand HLA-G inhibits immune activation, resulting in indirect promotion of tumor development ${ }^{[8]}$. LILRB1 modulates the differentiation and function of dendritic cells, resulting in poor stimulating activity for primary and memory $T$ cell proliferation ${ }^{[9]}$. LILRB1 also exhibited immune inhibition on NK cells ${ }^{[10]}$. Although the ligand for LILRB3 is not so clear, some studies suggested that LILRB3 was associated with cytokeratin-associated proteins exposed on necrotic cancer cells and might involve in altering the immune responses within the tumor microenvironment ${ }^{[11]}$. LILRB5 is considered to be an orphan receptor but may be associated with mycobacteria to subvert immune responses ${ }^{[12 ; 13]}$. In addition to expression on immune cells, the LILRB family was also found to be expressed in multiple malignant cells, such as liver cancer and lung cancer $^{[14 ; 15]}$. Cheng et $a^{[16]}$ found that LILRB1 in hepatocarcinoma cells might integrate with SHP1 to exert an antitumor effect in liver cancer patients. In contrast, some studies have also shown that LILRB2 regulates tumor cell proliferation, invasion, and migration and promotes tumor progression in lung cancer ${ }^{[17 ; 18]}$. LILRB4 was specific expressed on leukemia cells in monocytic acute myeloid leukemia but not in myelomonocytic acute myeloid leukemia ${ }^{[19]}$.

Therefore, the researches about LILRB family members in cancer tissues are limited and the function of these is not clear. Moreover, the expression levels of the LILRB family and their correlation with clinical features and prognosis have not been reported completely, especially using bioinformatics analysis.

Therefore, in this study, we first used the Oncomine and GEPIA databases to analyze the expression levels of the LILRB family in liver tumor tissues and their relationship with liver tumor stages. Moreover, immunohistochemistry was used to verify the results. Next, Kaplan-Meier Plotter was used to assess whether the LILRB family can be used to predict the prognosis of liver cancer patients. Then, we analyzed the correlation between LILRB family expression and immune cell infiltration by using TIMER and TISIDB. Finally, the retrieval of interacting genes with the LILRB family was exploded by using STRING, and the potential signaling pathways related to the LILRB family were predicted through KEGG analysis.

\section{Materials And Methods}

\subsection{Oncomine database}

The Oncomine platform (www.oncomine.org) is an online cancer microarray database containing 715 datasets and 86,733 samples. We used the Oncomine database to analyze the transcriptional levels of the LILRB family in different cancers, especially liver cancer. The conditions of the search filter were as 
follows: LILRB family gene name, cancer vs. normal analysis, threshold $p$-value, 0.05 , threshold fold change, 1.5 , threshold gene rank, top $10 \%$, and data type mRNA.

\subsection{GEPIA database}

Gene expression profiling interactive analysis (GEPIA) (gepia.cancer-pku.cn) 2.0 was developed by the Zhang laboratory from Peking University ${ }^{[20]}$. This online database served to analyze the RNA sequencing expression data of 9,736 tumors and 8,587 normal samples from the TCGA and GTEx projects.

Tumor/normal differential expression and profiling according to pathological stages could be analyzed in GEPIA 2.0 in this study.

\subsection{Kaplan-Meier Plotter}

The Kaplan-Meier plotter (kmplot.com/analysis/) could assess the effect of genes (mRNA, miRNA, and protein) on survival in 21 cancer types, including breast, ovarian, lung, gastric and liver cancer ${ }^{[21]}$. We used this database to analyze the correlation between LILRB family mRNA expression and the overall survival (OS), relapse-free survival (RFS), progression-free survival (PFS) and disease-free survival (DSS) of liver cancer patients. We also used this database to analyze the relationship between LILRB family mRNA expression and different tumor stages and risk factors and the prognosis of liver cancer patients. We chose to select the best cutoff to split the LILRB family into a low expression cohort and a high expression cohort.

\subsection{Timer}

Tumor immune estimation resources (Timer) (cistrome.shinyapps.io/timer/) served as a comprehensive resource for the systematic analysis of immune infiltrates across diverse cancer types ${ }^{[22]}$. The abundances of six immune infiltrates, $\mathrm{B}$ cells, $\mathrm{CD} 8^{+} \mathrm{T}$ cells, $\mathrm{CD} 4^{+} \mathrm{T}$ cells, neutrophils, macrophages and dendritic cells were estimated by the TIMER algorithm. We used this database to analyze the relationship between LILRB family expression and tumor infiltrating lymphocytes (TILs).

\subsection{TISIDB}

TISIDB (cis.hku.hk/TISIDB/) was developed by the Zhang laboratory from the University of Hong Kong in $2019^{[23]}$. TISIDB is a web portal for tumor and immune cell interactions and integrates multiple heterogeneous data types, including the PubMed database and The Cancer Genome Atlas (TCGA). We used this web portal to assess the relationship between LILRB family expression and various kinds of TIL subsets.

\section{6 cBioportal}

The cBioPortal (www.cbioportal.org) is an online tool to explore, to visualize, and to analyze multidimensional cancer genomics data ${ }^{[24 ; 25]}$. We used this website to analyze the mutations in LILRB family genes and the correlation between each LILRB family member and mRNA expression.

\subsection{STRING database}


STRING (string-db.org/cgi/input.pl) is an online database of known and predicted protein-protein interactions (PPIs) ${ }^{[26]}$. The sources of PPIs in STRING contain genomic context predictions, highthroughput lab experiments, coexpression, automated text mining and previous knowledge in databases. STRING was used to construct a PPI network of the LILRB family. The condition of the minimum required interaction score was the highest confidence (0.900). Then, Cytoscape software V3.8.0 was employed to analyze the relationship between LILRB family members and their related genes.

\subsection{DAVID database}

The Database for Annotation, Visualization and Integrated Discovery (DAVID) database (david.ncifcrf.gov/home.jsp) was used to carry out gene ontology (GO) and Kyoto Encyclopedia of Genes and Genomes (KEGG) analysis ${ }^{[27]}$. $\mathrm{R}$ language was used to process the results. A false discovery rate (FDR) $<0.05$ was considered to be significant.

\subsection{Patients and samples}

Ten diagnostic liver cancer patients were included in this study. Primary liver cancer tissues and their corresponding tumor-free liver tissues (TFLs) were obtained from surgical resection. The clinical information of these ten diagnostic liver cancer patients was shown in table.1. This study was approved by the medical ethical committee of the Second Hospital of Nanjing, Nanjing University of Chinese Medicine. Written informed consent was obtained from all donors in accordance with the Declaration of Helsinki (1964) before tissue sample collection.

\subsection{Immunohistochemistry}

Paraffin-embedded liver cancer tissues were sectioned into 3- $\mu \mathrm{m}$ pieces. Tumor sections were incubated with rabbit polyclonal antibodies against LILRB1, LILRB2, LILRB3, LILRB4 and LILRB5 at $4{ }^{\circ} \mathrm{C}$ for 12 hours. The antibodies against LILRB1, LILRB3, LILRB4 and LILRB5 were from Abcam (product code: ab229186, ab271287, ab229747, ab121357) and the antibody against LILRB2 was from Invitrogen (product code: PA5-103913). To detect primary antibody binding, the sections were conjugated with goat anti-rabbit IgG H\&L (HRP) (Abcam, Cambridge, UK) or goat anti-rabbit lgG (HRP) (Invitrogen, Carlsbad, CA, USA) at $25{ }^{\circ} \mathrm{C}$ for 2 hours. Finally, the tumor sections were visualized with 3,3-diaminobenzidine and counterstained with hematoxylin. The tumor sections were observed by light microscopy.

\subsection{Isolation of single cells and flow cytometry analysis}

Liver tumor tissues and TFL were cut into approximately 1- $\mathrm{mm}^{3}$ pieces and digested with $0.125 \mathrm{mg} / \mathrm{ml}$ collagenase IV (Sigma-Aldrich, St. Louis, MO) and $0.2 \mathrm{mg} / \mathrm{ml}$ DNAse I (Roche, Indianapolis, IN) in Hanks solution for 1 hour. Then, cells were isolated by lttration of liver slurry through $40-\mu \mathrm{m}$ Itters (BD Biosciences, San Jose, CA, USA), followed by centrifugation at $400 \mathrm{~g}$ for $10 \mathrm{~min}$. These cells were washed twice with PBS ( $\mathrm{pH}=7.4$ ) with 1\% FBS (Invitrogen, Carlsbad, CA, USA). Next, cells were labeled with monoclonal antibodies, including CD3-PerCp (OKT3), CD56-PeCy7 (MEM-188), CD16-APC-Cy7 (CB16) and LIVE/DEAD ${ }^{\circledR}$ Aqua, for $30 \mathrm{~min}$ at $4{ }^{\circ} \mathrm{C}$ (Thermo Fisher Scientific, Waltham, MA, USA). After washing twice 
in PBS with 1\% FBS, cells were analyzed by a FACS Canto II flow cytometer (BD Biosciences, San Jose, CA, USA).

\subsection{Statistical Analysis}

Data are expressed as the mean \pm SEM and were analyzed by Student's $t$-test using GraphPad 6 . Significance was defined as $<0.05$.

\section{Results}

\subsection{Transcriptional levels of the LILRB family in patients with liver cancer}

The LILRB family contains five subtypes: LILRB1, LILRB2, LILRB3, LILRB4 and LILRB5. We compared the mRNA expression of the LILRB family in different cancers with that in corresponding normal tissues based on the Oncomine database. As shown in Fig. 1, compared with that of normal tissues, in addition to LILRB4, other LILRB family members were downregulated in liver tumor tissues. All the data came from four datasets, and all the tumor data came from $\mathrm{HCC}^{[28-31]}$. Three of the datasets had the same results even if two datasets did not achieve the condition of fold change $>1.5$, while the Mas`s dataset had the opposite results (Table.2).

The table shows that (1) the transcriptional levels of LILRB1 were significantly downregulated in HCC patients in three datasets with fold changes of $-1.602,-1.251$, and -1.182 ; (2) compared with normal tumors, LILRB2 was also downregulated in liver tumors in three datasets with fold changes of -2.098 , $-1.441,-1.281$, and -1.708 ; (3) compared to normal tissues, LILRB3 was found to be decreased in HCC with fold changes of $-2.078,-1.391,-1.341$, and -1.322 ; (4) while the results from the other three datasets showed that there was no difference in LILRB4 expression between liver tumors and normal tissues, LILRB4 was upregulated in liver tumors in Mas's datasets with fold changes of 1.7; and (5) compared with normal tissues, LILRB5 expression was significantly decreased in HCC patients in three datasets with fold changes of $-1.656,-2.826$, and -1.321 , respectively.

To verify the above results further, GEPIA 2.0 was used to compare the mRNA expression of the LILRB family between liver tumor tissues and normal tissues. The LIMMA method was used to compare liver tumor tissues and their paired normal samples regarding mRNA expression of the LILRB family. The results showed that LILRB1, LILRB2, LILRB3, and LILRB5 were downregulated, and compared with liver normal tissues, LILRB4 expression was upregulated in liver tumor tissues, which was consistent with the results in the Oncomine database (Fig. 2).

To assess LILRB expression at the protein level, we detected LILRB family expression in liver tumors and their corresponding TFLs by using immunohistochemistry. We found that the expression of LILRB1, LILRB2, LILRB3 and LILRB5 was lower in liver tumors than in TFL, while the expression of LILRB4 was high in liver tumors than in TFL (Fig. 3). 


\subsection{The relationship between the LILRB family and tumor stage in liver cancer patients}

We compared the relationship between LILRB family expression and four tumor stages in liver cancer patients by using GEPIA 2.0. One-way ANOVA was used to analyze these results. In agreement with the literature ${ }^{[16]}$, although there was no significant correlation between LILRB family expression and tumor stage in liver cancer patients, compared with that in other stages of liver cancer patients, the expression of the LILRB family in stage IV liver cancer patients decreased (Fig. 4).

\subsection{Prognostic value of LILRB family mRNA expression in all liver cancers}

Kaplan-Meier Plotter was used to examine the prognostic value of LILRB family mRNA expression levels in all liver cancers. We compared the correlation between mRNA expression of LILRB family members and OS, RFS, PFS and DSS of liver cancer patients. The results revealed that patients in LILRB2 and LILRB5 low group had shortened OS and DSS and low expression of all LILRB family members predicted poorer patients RFS and PFS ( $p<0.05)$ (Fig. 5). Median survival times of LILRB high groups in OS, PFS, PFS and DSS were longer than these of LILRB low groups, when there was statistical difference between these two groups (Table.3).

Next, we further investigated the prognostic value of LILRB family mRNA expression in liver cancer with different histologic stages by using Kaplan-Meier Plotter. The results were as follows: (1) The high mRNA

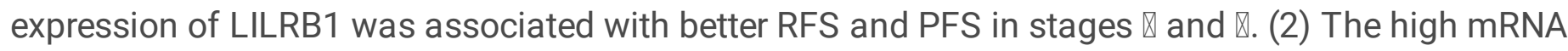

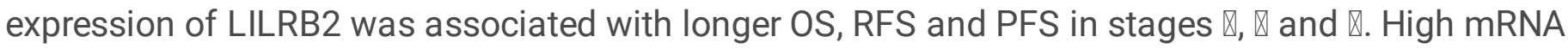
expression of LILRB2 was predicted to have better DSS in stages $\nabla$ and $\nabla$. (3) High expression of LILRB3 mRNA was correlated with better RFS and PFS in stage $\triangle$, while low expression of LILRB3 mRNA was associated with better DSS in stage $\mathbb{~}$. (4) The low mRNA expression of LILRB4 was associated with

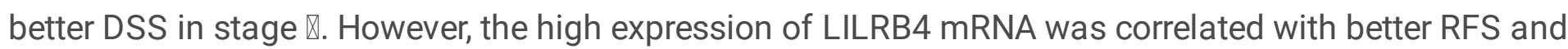
PFS in stage $\triangle$ as well as better PFS in stage $\triangle$. (5) High mRNA expression of LILRB5 was predicted to be

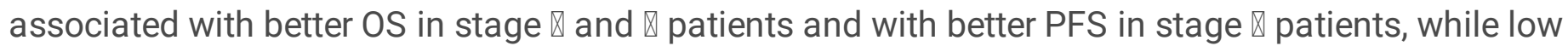
mRNA expression of LILRB5 was associated with better OS in stage $₫$ patients (Supplementary Table S1).

\subsection{Prognostic value of LILRB mRNA expression with risk factors in liver cancer}

The two main risk factors, alcohol consumption and hepatitis virus, could induce liver cancer. Therefore, we compared the relationship between LILRB mRNA expression and these two risk factors by using Kaplan-Meier Plotter. Regarding alcohol consumption, we summarized the following: (1) The high mRNA expression of LILRB1 was associated with better OS and DSS in liver cancer patients in alcoholconsuming cohorts and better RFS and PFS in liver cancer patients in nonalcohol-consuming cohorts. (2) In liver cancer with alcohol consumption cohorts, high LILRB2 mRNA expression was correlated with 
better OS, RFS, PFS and DSS. The liver cancer patients with no alcohol consumption habit who expressed high LILRB mRNA had better RFS, PFS and DSS. (3) The high mRNA expression of LILRB3 was associated with better RFS in liver patients in nonalcohol-consuming cohorts. (4) The high mRNA expression of LILRB4 was only correlated with better RFS and PFS in liver cancer patients in alcohol consumption cohorts and better RFS in liver cancer patients in nonalcohol-consuming cohorts. (5) Regardless of whether liver cancer patients drink, the high mRNA expression of LILRB5 in these two kinds of patients was predicted to have better OS, RFS, PFS and DSS (Supplementary Table S2).

The results of the relationship between mRNA expression of the LILRB family and different hepatitis virus infection statuses are shown in Supplementary Table S3. Regarding hepatitis virus-infected liver cancer patients, the results showed that (1) the high mRNA expression of LILRB2 was associated with better RFS and PFS; (2) the high mRNA expression of LILRB3 and LILRB5 was correlated with better PFS; and (3) the low mRNA expression of LILRB4 was predicted to have better OS and DSS. In contrast, the high mRNA expression of LILRB4 was associated with better RFS and PFS. Regarding nonhepatitis virus-infected liver cancer patients, we concluded that high mRNA expression of LILRB1, LILRB2 and LILRB5 was associated with better OS, RFS, PFS and DSS, and that high LILRB4 mRNA expression was correlated with better RFS.

\subsection{The relationship between LILRB family expression and immune cell infiltration}

The LILRB family is considered to be an immune inhibitory receptor. TILs are associated with prognostic indicators for liver cancer ${ }^{[32]}$. Therefore, we speculated that there was a correlation between LILRB family expression and TIL infiltration in liver cancer patients. Timer database was used to analyze the suspected association. The results showed that all LILRB family members were significantly associated with tumor purity, $\mathrm{B}$ cells, $\mathrm{CD} 8^{+} \mathrm{T}$ cells, $\mathrm{CD} 4^{+} \mathrm{T}$ cells, macrophages, neutrophils and dendritic cells (Fig. 6).

The TISIDB database was used to assess the relationship between LILRB family expression and TIL subsets. Although there was no significant correlation of LILRB5 expression with activated $\mathrm{CD} 4^{+} \mathrm{T}$ cells and CD56 dim natural killer cells, the expression of other LILRB family members in liver cancer was strongly associated with various TIL subsets (Supplementary Tables S4-S8). Together these Indings suggested that the LILRB family plays an important role in recruitment and regulation of immune in $\square$ ltrating cells in liver cancer.

\subsection{The mutations of LILRB family genes}

The cBioPortal online tool was used to analyze the mutations of LILRB family genes. The main mutation types of LILRB family genes in liver cancers were missense mutations and amplifications (Fig. 7A). The mutations were concentrated in hepatocellular carcinoma and hepatobiliary cancer. A total of $5.42 \%$ of LILRB genes in 369 cases of hepatocellular carcinoma were altered, and 3.38\% of LILRB genes in 916 cases of hepatobiliary cancer were altered (Fig. 7B). 
Then, we analyzed the correlation between each LILRB member and their mRNA expression by using the cBioPortal online tool. The statistical method was Spearman. The results showed that there was a significant relationship between each LILRB member (Table.4) $(p<0.05)$.

\subsection{Predicted functions and pathways of the LILRB family in liver cancer}

To understand the biological significance and consequences of the protein profiling of the LILRB family, we constructed a PPI network by using STRING. The Cytoscape software was used to process it. As shown in Fig. 8, PPI analysis showed known and predicted interactions between LILRB family members and 50 proteins.

Next, GO and KEGG analyses for the LILRB family and these 50 genes were performed using the DAVID online tool. The GO analysis showed that there were 38 items of biological process (BP), 25 items of cellular component (CC) and 23 items of molecular function (MF) with FDR less than 0.05. The first 10 items were used to plot. The GO analysis for BP showed that LILRB family members and their related genes were mostly enriched in the immune response and antigen processing and presentation of antigens (Fig. 9A). The GO analysis for CC revealed that LILRB family members and their related genes were significantly enriched in the plasma membrane and cell surface (Fig. 9B). The GO analysis for MF showed that LILRB family members and their related genes were associated with beta-2-microglobulin binding, peptide antigen binding and MHC class I protein binding (Fig. 9C). In KEGG analysis, 27 pathways were related to the function of LILRB family members (Fig. 9D). Among them, antigen processing and presentation, cell adhesion molecules (CAMs), phagosomes, natural killer cell-mediated cytotoxicity, and leukocyte transendothelial migration were involved in antitumor immunity in liver cancer.

Based on the KEGG results and previous researches ${ }^{[33-35]}$, we hypothesized that LILRB family members and their related genes are involved in the pathways of antigen processing and presentation and natural killer cell-mediated cytotoxicity (Fig. 10A and 10B).

Our previous study verified that compared with their corresponding tumor-free liver tissues (TFLs), the percentage of infiltrating CD1 $c^{+}$myeloid DCs (mDCs) was significantly decreased in liver tumor tissues [7]. Considering that infiltrating NK cells in liver cancer might be involved in the signaling of the LILRB family, we further compared the percentage of NK cells in liver tumor tissues and the corresponding TFL. Human NK cells can be segregated into three major subsets: CD56 ${ }^{\text {bright }}$ CD16 ${ }^{\mathrm{dim} /-}, \mathrm{CD}^{\mathrm{dim}} \mathrm{CD}^{\mathrm{di}}{ }^{+}$, CD56 ${ }^{\mathrm{dim}} \mathrm{CD} 16^{\mathrm{dim} /-} \mathrm{NK}$ cells. The strategy of gating these three NK cells subsets were according to the literature ${ }^{[36]}$. The results shown that the percentage of $C D 56^{\text {bright }} C D^{\text {dim/- }} \mathrm{NK}$ cell in liver tumor tissues was lower than that from TFL tissues (Tumor vs TFL: $30.84 \% \pm 7.77 \%$ vs $43.85 \% \pm 4.12 \%, p<0.05$ )

(Fig. 11). In contrast, the percentage of CD $56^{\operatorname{dim}}$ CD $16^{+} \mathrm{NK}$ cell in liver tumor tissues was higher than that from TFL tissues (Tumor vs TFL: $49.98 \% \pm 8.66 \%$ vs $29.73 \% \pm 4.78 \%, p<0.05$ ).

\section{Discussion}


The LILRB family is considered to be an immune inhibitory receptor. Most studies have focused on the expression of the LILRB family on immune cells in many cancers ${ }^{[19 ; 37]}$. A recent study showed that LILRB2 was expressed in lung cancer tissues and that its expression resulted in poor patient $\mathrm{OS}^{[38]}$. On the contrary, Cheng et $a^{[16]}$ found that LILRB1 in hepatocarcinoma cells might integrate with SHP1 to exert an antitumor effect in liver cancer patients. Hence, the expression of LILRB family members have observed in tumor cells with no clear function. Moreover, the expression of all LILRB family members and their relationships with prognosis in liver cancer patients have yet to be determined. In this study, a series of bioinformatics analysis methods and experiments were used to explode the expression, prognosis and potential function of the LILRB family in liver cancer.

First, we assessed the expression of the LILRB family in liver tumor tissues and its correlation with the survival of liver cancer patients. The Oncomine and GEIPA 2.0 databases confirmed that LILRB1, LILRB2, LILRB3, and LILRB5 expression was lower in liver tumor tissues than in normal tissues and that LILRB4 was overexpressed in liver tumor tissues. To further verify these results, we assessed LILRB family member expression at the protein level by using immunohistochemistry. The results were in accordance with the mRNA expression of the LILRB family, which was also consistent with a previous study ${ }^{[16]}$. In contrast, some studies proved that LILRB2 was expressed at higher levels in HCC tissues, which needs further verification with large samples in future studies ${ }^{[38 ;}$ 39]. In addition, we also used Kaplan-Meier Plotter to explode the prognostic merit of LILRB family member expression in liver cancer. Our results showed that LILRB2 and LILRB5 expression was significantly associated with OS and DSS and that the mRNA expression of all LILRB family members was significantly correlated with RFS and PFS in liver cancer patients. Hence, these results robustly indicated that the LILRB family was a potential prognostic biomarker in liver cancer patients.

Numerous studies have proven that the LILRB family plays an immunosuppressive role in the immune system. These immune inhibitory receptors could restrict $T$ cell infiltration and killing ability, leading to impaired antitumor responses ${ }^{[40]}$. Scientists have discovered that PIR-B is expressed on murine $B$ cells and myeloid cells, as the ortholog for human LILRB1/B2, blocked the access of CD8aa to MHC-I, resulting in weaker antitumor immunity ${ }^{[41]}$. Researchers have also found that disruption of either $\mathrm{MHC}$ class I or LILRB1 enhanced phagocytosis of tumor cells in macrophages ${ }^{[37]}$. For these reasons, we compared the correlation between the LILRB family and infiltrating immune cells. The mRNA expression of the LILRB family was inversely proportional to the purity of the liver tumor, while LILRB family expression was proportional to immune cells, including $B$ cells, $C D 8^{+} T$ cells, $C D 4^{+} T$ cells, macrophages, neutrophils and dendritic cells. The correlation between LILRB family members expression and immune cells implicated the role of LILRBs in recruiting and regulating tumor immunology in liver cancer.

Previous studies have explored some mechanisms of the LILRB family expressed on immune cells in the immune system. Khanolkar et al found that LILRB1 on dendritic cells increased the expression of the NF$K B$ regulator $A B I N 1 / T N I P 1$, resulting in suppression of the stimulatory effect of dendritic cells on $T$ cells. Research has also discovered that LILRB2 expressed on tumor-associated myeloid cells inhibited the 
activation of SHP1/2, AKT and STAT6, leading to restraint of the function of M1-like macrophages and promotion of the function of M2-like macrophages ${ }^{[42]}$. However, these mechanisms are concerned with the expression of the LILRB family on immune cells, and the mechanisms of the LILRB family expressed in the tumor microenvironment, especially on solid tumors, have not yet been discovered clearly. We identified 50 potential and validated proteins that interacted with the LILRB family by using STRING. The KEGG analysis results and previous experiments showed that the LILRB family might be involved in antigen processing and presentation and NK cell pathways ${ }^{[43]}$. NK cells have been studied as three separate populations: CD56 ${ }^{\text {bright }} C D 16^{\mathrm{dim} /-}, \mathrm{CD} 56^{\mathrm{dim}} \mathrm{CD} 16^{+}, \mathrm{CD} 56^{\mathrm{dim}} \mathrm{CD} 16^{\mathrm{dim} /-} \mathrm{NK}$ cells.

CD56 ${ }^{\text {bright }}$ CD $16^{\text {dim/- }}$ NK cells secreted cytokines to kill tumor cells and regulate the immune system. CD56 ${ }^{\text {dim }}$ CD $16^{+}$NK cells primarily demonstrated cytotoxicity effect. CD $56^{\text {dim }}$ CD $16^{\text {dim/- }}$ NK cells might be the immediate precursors of the $\mathrm{CD} 56^{\mathrm{dim}} \mathrm{CD} 16^{+}$subset, which remained to be clarified in the future $\mathrm{e}^{[44]}$. Consistent with the literature, $\mathrm{CD} 56^{\mathrm{dim}} \mathrm{CD} 16^{+} \mathrm{NK}$ cells were significantly reduced in liver tumor tissues compared with TFL, resulting in secreting less cytokines that killed tumor cells ${ }^{[45]}$. This might due to the reduced expressed LILRB family members on tumor cells, leading to recruiting less NK cells. However, we don't deeply study the effect of LILRB expression in liver tumors on NK cell function, and we will study its mechanism in the future.

\section{Conclusions}

In this study, using bioinformatics analysis, we identified decreases in LILRB1, LILRB2, LILRB3 and LILRB5 and increases in LILRB4 in liver tumor tissues, which were confirmed at the mRNA and protein levels. Moreover, survival analysis revealed that LILRB2 and LILRB5 mRNA levels were significantly associated with OS and DSS and that the mRNA expression of all LILRB family members was significantly correlated with RFS and PFS. High expression of LILRB family members was associated with increased infiltration of immune cells, including $B$ cells, $C D 8^{+} T$ cells, $C D 4^{+} T$ cells, macrophages, neutrophils and dendritic cells. Finally, PPI prediction analysis of the LILRB family and KEGG analysis suggested that LILRB family members might be involved in antigen processing and presentation and natural killer cell pathways during the development of liver cancer.

\section{Declarations}

\section{Ethics approval and consent to participate}

This study was approved by the medical ethical committee of the Second Hospital of Nanjing, Nanjing University of Chinese Medicine. Written informed consent was obtained from all donors in accordance with the Declaration of Helsinki (1964) before tissue sample collection.

\section{Consent for publication}

Not applicable 


\section{Availability of data and materials}

The datasets used and/or analyzed during the current study are available from the corresponding author on reasonable request.

All the databases used in this study were below:

Oncomine database: www.oncomine.org

GEPIA database: http://gepia2.cancer-pku.cn/\#index

Kaplan-Meier Plotter: http://kmplot.com/analysis/index.php?p=service\&cancer=liver_rnaseq

Timer: https://cistrome.shinyapps.io/timer/

TISIDB: http://cis.hku.hk/TISIDB/

cBioportal: http://www.cbioportal.org

STRING database: https://string-db.org/cgi/input.pl

DAVID database: https://david.ncifcrf.gov/summary.jsp

\section{Competing interests}

The authors declare that they have no competing interests.

\section{Funding}

This work was supported by Science and Technology Commission of Nanjing [grant number 201803001] and Medical Science and Technology Development Foundation, Nanjing Department of Health [grant number YKK17167].

\section{Authors' contributions}

L.L.W. designed the experiments and wrote the paper. M.C., J.K.Z. and J.Y.L. collected the data. F.N.S. and A.D.G. collected the clinical samples. D.D.Y. analyzed the data. J.F. and Y.X.Y. conducted the experiments and edited the paper.

\section{Acknowledgements}

Not applicable.

\section{References}


1. Bray F, Ferlay J, Soerjomataram I, et al. Global cancer statistics 2018: GLOBOCAN estimates of incidence and mortality worldwide for 36 cancers in 185 countries[J]. CA Cancer J Clin. 2018;68(6):394-424.

2. Mohammadian M, Soroush A, Mohammadian-Hafshejani A, et al. Incidence and Mortality of Liver Cancer and Their Relationship with Development in Asia[J]. Asian Pac J Cancer Prev. 2016;17(4):2041-7.

3. Mak D, Sengayi M, Chen WC, et al. Liver cancer mortality trends in South Africa: 1999-2015[J]. BMC Cancer. 2018;18(1):798.

4. Feng R-M, Zong Y-N, Cao S-M, et al. Current cancer situation in China: good or bad news from the 2018 Global Cancer Statistics?[J]. Cancer Commun. 2019;39(1):22.

5. Zhou J, Sun H-C, Wang Z, et al. Guidelines for Diagnosis and Treatment of Primary Liver Cancer in China (2017 Edition)[J]. Liver cancer. 2018;7(3):235-60.

6. Jones DC, Kosmoliaptsis V, Apps R, et al. HLA Class I Allelic Sequence and Conformation Regulate Leukocyte Ig-Like Receptor Binding[J]. J Immunol. 2011;186(5):2990.

7. Wang L, Fan J, Ye W, et al. The Expression of ILT4 in Myeloid Dendritic Cells in Patients with Hepatocellular Carcinoma[J]. Immunological Investigations, 2019: 1-15.

8. Burshtyn DN, Morcos C. The Expanding Spectrum of Ligands for Leukocyte Ig-like Receptors[J]. J Immunol. 2016;196(3):947.

9. Young NT, Waller ECP, Patel R, et al. The inhibitory receptor LILRB1 modulates the differentiation and regulatory potential of human dendritic cells[J]. Blood. 2008;111(6):3090-6.

10. Chen $\mathrm{H}$, Chen $\mathrm{Y}$, Deng $\mathrm{M}$, et al. Antagonistic anti-LILRB1 monoclonal antibody regulates antitumor functions of natural killer cells[J]. J immunother Cancer. 2020;8(2):e000515.

11. Jones DC, Hewitt CRA, López- Ivarez MR, et al. Allele-specific recognition by LILRB3 and LILRA6 of a cytokeratin 8 - associated ligand on necrotic glandular epithelial cells[J]. Oncotarget, 2016, 7(13).

12. Hogan LE, Jones DC, Allen RL. Expression of the innate immune receptor LILRB5 on monocytes is associated with mycobacteria exposure[J]. Sci Rep. 2016;6(1):21780.

13. Yeboah M, Papagregoriou C, Jones DC, et al. LILRB3 (ILT5) is a myeloid cell checkpoint that elicits profound immunomodulation[J]. JCl insight. 2020;5(18):e141593.

14. Kang X, Kim J, Deng M, et al. Inhibitory leukocyte immunoglobulin-like receptors: Immune checkpoint proteins and tumor sustaining factors[J]. Cell Cycle. 2016;15(1):25-40.

15. Wiśniewski A, Kowal A, Wyrodek E, et al. Genetic polymorphisms and expression of HLA-G and its receptors, KIR2DL4 and LILRB1, in non-small cell lung cancer[J]. Tissue Antigens. 2015;85(6):46675 .

16. Cheng J, Luan J, Chen P, et al. Immunosuppressive receptor LILRB1 acts as a potential regulator in hepatocellular carcinoma by integrating with SHP1[J]. Cancer Biomarkers. 2020;28:309-19.

17. Liu X, Yu X, Xie J, et al. ANGPTL2/LILRB2 signaling promotes the propagation of lung cancer cells[J]. Oncotarget. 2015;6(25):21004-15. 
18. Zhang P, Guo X, Li J, et al. Immunoglobulin-like transcript 4 promotes tumor progression and metastasis and up-regulates VEGF-C expression via ERK signaling pathway in non-small cell lung cancer[J]. Oncotarget. 2015;6(15):13550-63.

19. John S, Chen H, Deng M, et al. A Novel Anti-LILRB4 CAR-T Cell for the Treatment of Monocytic AML[J]. Mol Ther. 2018;26(10):2487-95.

20. Tang Z, Li C, Kang B, et al. GEPIA: a web server for cancer and normal gene expression profiling and interactive analyses[J]. Nucleic acids research. 2017;45(W1):W98-102.

21. Nagy, Lánczky A, Menyhárt 0 , et al. Validation of miRNA prognostic power in hepatocellular carcinoma using expression data of independent datasets[J]. Scientific reports. 2018;8(1):9227-7.

22. Li T, Fan J, Wang B, et al. TIMER: A Web Server for Comprehensive Analysis of Tumor-Infiltrating Immune Cells[J]. Cancer research. 2017;77(21):e108-10.

23. Ru B, Wong CN, Tong Y, et al. TISIDB: an integrated repository portal for tumor-immune system interactions[J]. Bioinformatics. 2019;35(20):4200-2.

24. Cerami E, Gao J, Dogrusoz U, et al. The cBio Cancer Genomics Portal: An Open Platform for Exploring Multidimensional Cancer Genomics Data[J]. Cancer Discov. 2012;2(5):401.

25. Gao J, Aksoy BA, Dogrusoz U, et al. Integrative analysis of complex cancer genomics and clinical profiles using the cBioPortal[J]. Sci Signal. 2013;6(269):pl1-1.

26. Szklarczyk D, Gable AL, Lyon D, et al. STRING v11: protein-protein association networks with increased coverage, supporting functional discovery in genome-wide experimental datasets[J]. Nucleic Acids Res. 2018;47(D1):D607-13.

27. Huang DW, Sherman BT, Lempicki RA. Systematic and integrative analysis of large gene lists using DAVID bioinformatics resources[J]. Nat Protoc. 2009;4(1):44-57.

28. Wurmbach E, Chen Y-B, Khitrov G, et al. Genome-wide molecular profiles of HCV-induced dysplasia and hepatocellular carcinoma[J]. Hepatology. 2007;45(4):938-47.

29. Roessler S, Jia H-L, Budhu A, et al. A Unique Metastasis Gene Signature Enables Prediction of Tumor Relapse in Early-Stage Hepatocellular Carcinoma Patients[J]. Can Res. 2010;70(24):10202.

30. Mas VR, Maluf DG, Archer KJ, et al. Genes involved in viral carcinogenesis and tumor initiation in hepatitis C virus-induced hepatocellular carcinoma[J]. Mol Med. 2009;15(3-4):85-94.

31. Chen X, Cheung ST, So S, et al. Gene expression patterns in human liver cancers[J]. Molecular biology of the cell. 2002;13(6):1929-39.

32. Yao W, He J-C, Yang Y, et al. The Prognostic Value of Tumor-infiltrating Lymphocytes in Hepatocellular Carcinoma: a Systematic Review and Meta-analysis[J]. Sci Rep. 2017;7(1):7525.

33. Gu X, Laouar A, Wan J, et al. The gp49B1 Inhibitory Receptor Regulates the IFN-y Responses of T Cells and NK Cells[J]. J Immunol. 2003;170(8):4095.

34. Si YQ, Bian XK, Lu N, et al. Cyclosporine Induces Up-regulation of Immunoglobulin-like Transcripts 3 and 4 Expression on and Activity of NKL Cells[J]. Transplantation Proceedings, 2012, 44(5): 14071411. 
35. Jin Z, Fan J, Zhang Y, et al. Comparison of morphology, phenotypes and function between cultured human IL4DC and IFNDC[J]. Mol Med Rep. 2017;16(5):7345-54.

36. Vujanovic L, Chuckran C, Lin Y, et al. CD56dim CD16- Natural Killer Cell Profiling in Melanoma Patients Receiving a Cancer Vaccine and Interferon-a[J]. Frontiers in Immunology, 2019, 10(14).

37. Barkal AA, Weiskopf $\mathrm{K}$, Kao KS, et al. Engagement of MHC class I by the inhibitory receptor LILRB1 suppresses macrophages and is a target of cancer immunotherapy[J]. Nature immunology. 2018;19(1):76-84.

38. Li Q, Li J, Wang S, et al. Overexpressed immunoglobulin-like transcript (ILT) 4 in lung adenocarcinoma is correlated with immunosuppressive $T$ cell subset infiltration and poor patient outcomes[J]. Biomarker research. 2020;8:11-1.

39. Li X, Wei $X, X u H$, et al. Expression of leukocyte immunoglobulin-like receptor $B 2$ in hepatocellular carcinoma and its clinical significance[J]. J Cancer Res Ther. 2018;14(7):1655-9.

40. Thommen DS, Schumacher TN. T cell dysfunction in cancer[J]. Cancer cell. 2018;33(4):547-62.

41. Endo S, Sakamoto Y, Kobayashi E, et al. Regulation of cytotoxic T lymphocyte triggering by PIR-B on dendritic cells[J]. Proceedings of the National Academy of Sciences, 2008, 105(38): 14515-14520.

42. Chen H-M, Van Der Touw W, Wang YS, et al. Blocking immunoinhibitory receptor LILRB2 reprograms tumor-associated myeloid cells and promotes antitumor immunity[J]. J Clin Investig. 2018;128(12):5647-62.

43. Hoechst B, Voigtlaender T, Ormandy L, et al. Myeloid derived suppressor cells inhibit natural killer cells in patients with hepatocellular carcinoma via the NKp30 receptor[J]. Hepatology. 2009;50(3):799-807.

44. Amand M, Iserentant G, Poli A, et al. Human CD56dimCD16dim Cells As an Individualized Natural Killer Cell Subset[J]. Frontiers in Immunology, 2017, 8(699).

45. Cai L, Zhang Z, Zhou L, et al. Functional impairment in circulating and intrahepatic NK cells and relative mechanism in hepatocellular carcinoma patients[J]. Clinical Immunology. 2008;129(3):42837.

\section{Figures}




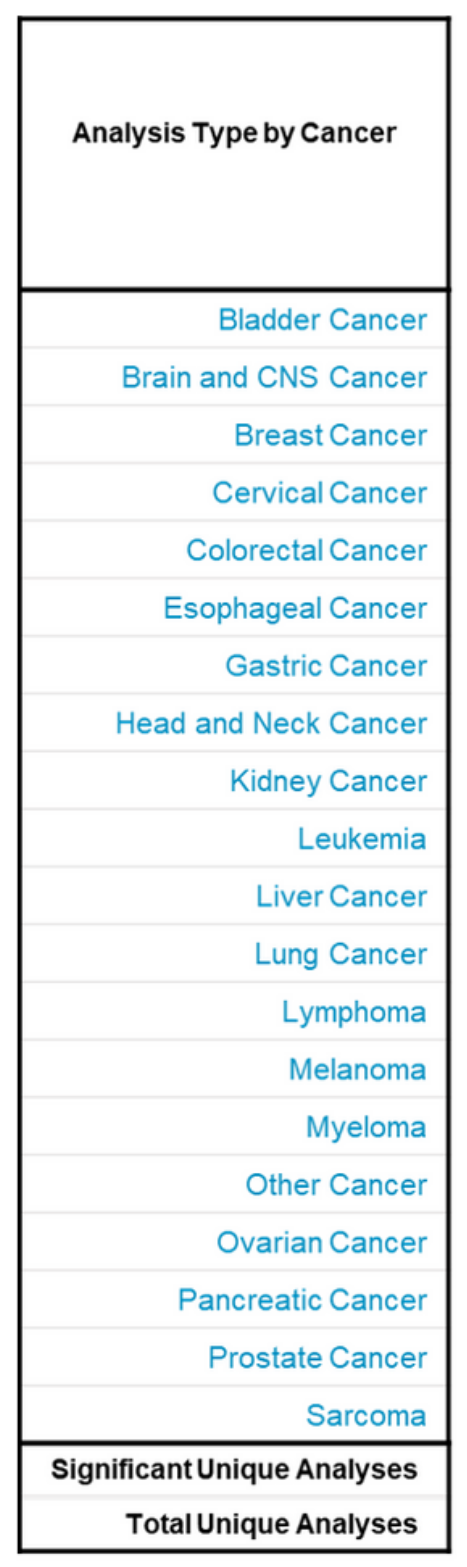

\begin{tabular}{|c|c|c|c|c|c|c|c|c|c|}
\hline \multicolumn{2}{|c|}{$\begin{array}{c}\text { Cancer } \\
\text { vs. } \\
\text { Normal } \\
\text { LILRB1 }\end{array}$} & \multicolumn{2}{|c|}{$\begin{array}{c}\text { Cancer } \\
\text { vs. } \\
\text { Normal } \\
\text { LILRB2 }\end{array}$} & \multicolumn{2}{|c|}{$\begin{array}{c}\text { Cancer } \\
\text { vs. } \\
\text { Normal } \\
\text { LILRB3 }\end{array}$} & \multicolumn{2}{|c|}{$\begin{array}{c}\text { Cancer } \\
\text { vs. } \\
\text { Normal } \\
\text { LILRB4 }\end{array}$} & \multicolumn{2}{|c|}{$\begin{array}{c}\text { Cancer } \\
\text { vs. } \\
\text { Normal } \\
\text { LILRB5 }\end{array}$} \\
\hline & & & 1 & & 1 & & & & 1 \\
\hline 4 & & 1 & 1 & 6 & & 3 & & & 1 \\
\hline \multirow[t]{5}{*}{4} & 8 & 9 & & 4 & 4 & 7 & 1 & 1 & 11 \\
\hline & & & & 1 & & & & & \\
\hline & 4 & & & & 1 & 1 & 1 & & 10 \\
\hline & & & & 2 & & 2 & & & \\
\hline & & 2 & 1 & 1 & & 5 & & & 2 \\
\hline 2 & 2 & 1 & & 1 & & 2 & 1 & & 1 \\
\hline 5 & 2 & 8 & & 3 & & 3 & & & \\
\hline \multirow[t]{3}{*}{6} & 3 & 3 & 7 & 2 & 8 & 3 & 1 & 1 & \\
\hline & 1 & & 1 & & 1 & 1 & & & 2 \\
\hline & 3 & & 5 & & 7 & & 1 & & 1 \\
\hline 7 & 3 & 6 & & 3 & & 10 & & & \\
\hline 1 & & & 1 & & & 1 & & & \\
\hline \multirow[t]{5}{*}{6} & 1 & 8 & & 2 & & 3 & 1 & 1 & \\
\hline & 1 & & & & & & & & \\
\hline & & & & & & & & & \\
\hline & & & 1 & & & & & & \\
\hline & 2 & 2 & 2 & & 4 & 1 & & & 1 \\
\hline 35 & 30 & 39 & 20 & 25 & 26 & 42 & 6 & 3 & 30 \\
\hline \multicolumn{2}{|c|}{309} & \multicolumn{2}{|c|}{324} & \multicolumn{2}{|c|}{301} & \multicolumn{2}{|c|}{317} & \multicolumn{2}{|c|}{278} \\
\hline
\end{tabular}

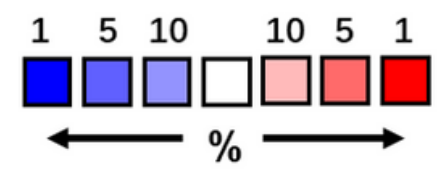

\section{Figure 1}

The transcription levels of the LILRB family in different types of human cancers. The figure was generated from the Oncomine database under the conditions of $p<0.05$, fold change $>1.5$, gene bank: top $10 \%$, data type: mRNA. Cell color is determined by the best gene rank percentile for the analyses within the cell. Blue represents underexpression and red represents overexpression. 

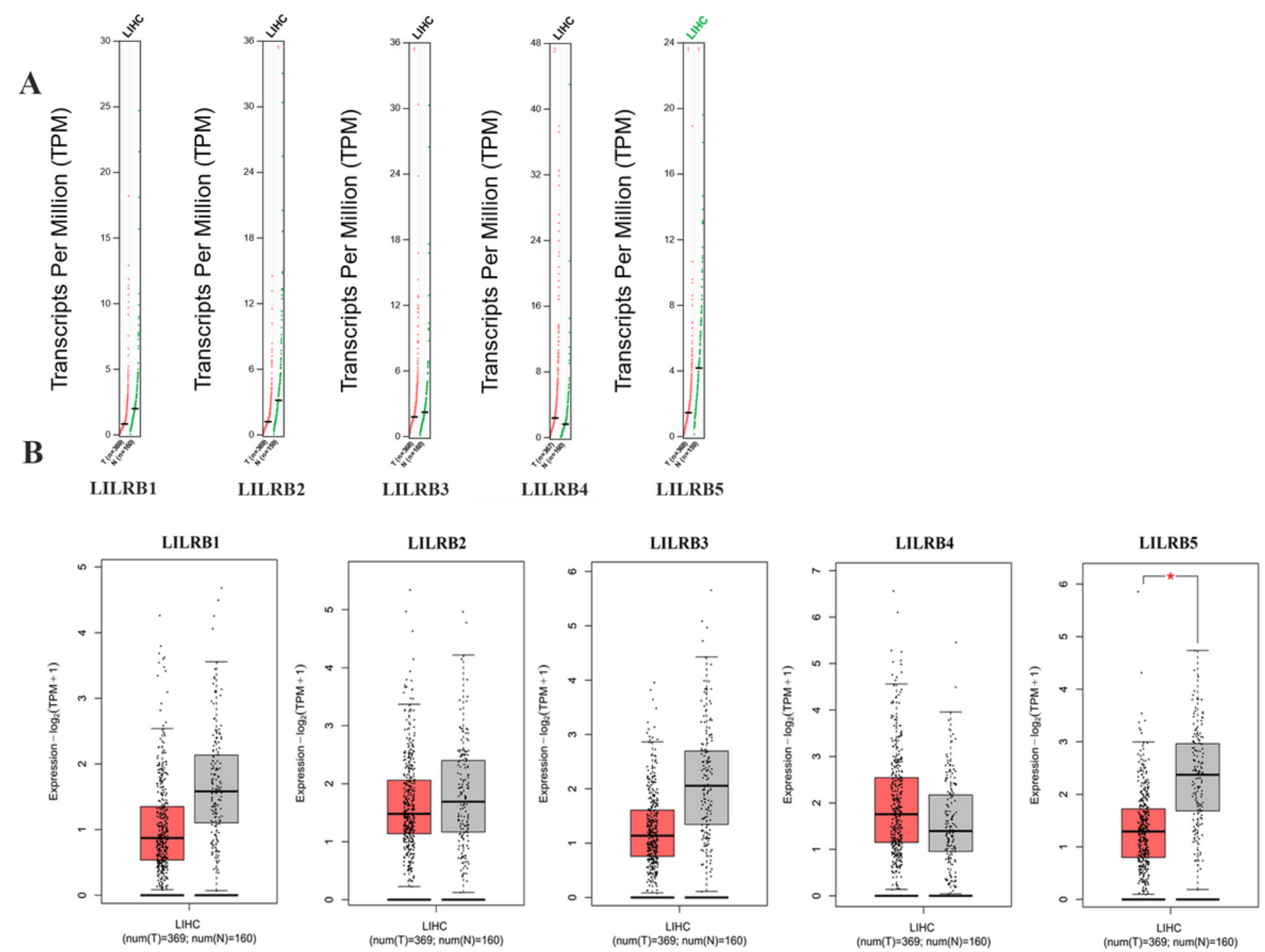

Figure 2

The transcription expression levels of the LILRB family in liver cancer (GEPIA 2.0). (A) Scatter diagram of individual LILRB member expression. The number of HCC tumor samples and normal tissues were noted in the figure. (B) Box plot of individual LILRB member expression. The red color represents tumor tissues, and the gray color represents normal tissues. The number of HCC tumor samples was 369 and the number of normal tissues was 160 . This figure was generated under the conditions of cutoff: 1, q-value cutoff: 0.01 . The ANOVA was used for tumor vs paired normal samples. $\left({ }^{\star} \mathrm{p}<0.05\right)$. 

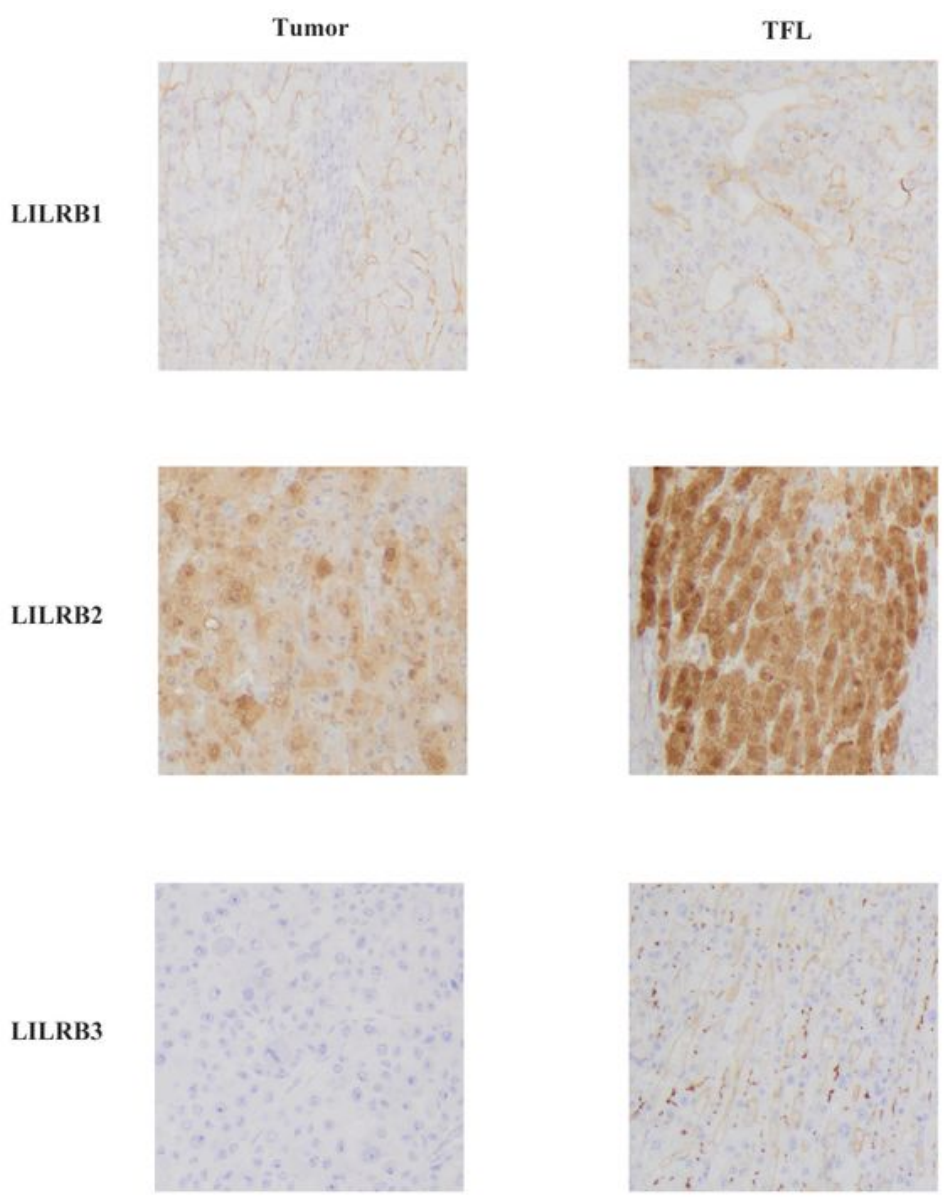

LILRB3
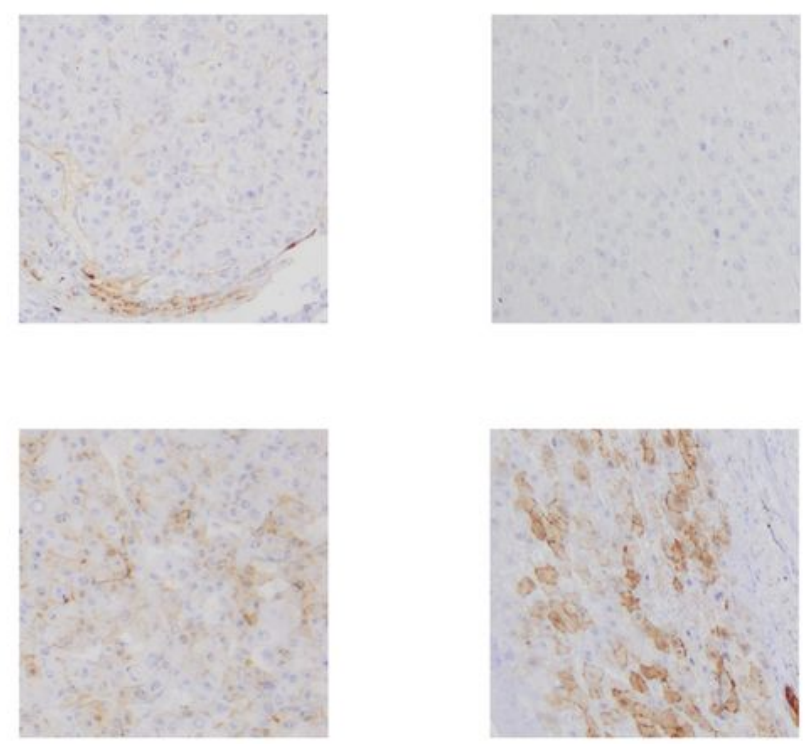

\section{Figure 3}

The expression of LILRB family members in liver cancer by immunohistochemical analysis.

Representative IHC images of LILRB family members expression in the microarray that contained 10 cases of liver tumor tissues and their TFL. Multiple images were taken and representative one was presented. Brown granules were defined as positive staining of LILRB family members. The magnification was $100 x$. 


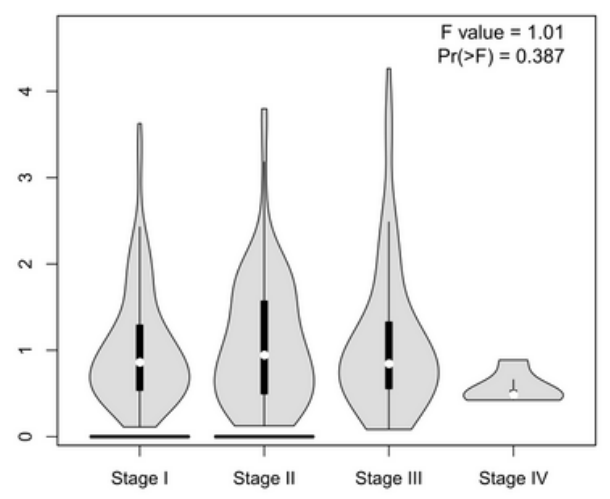

LILRB1

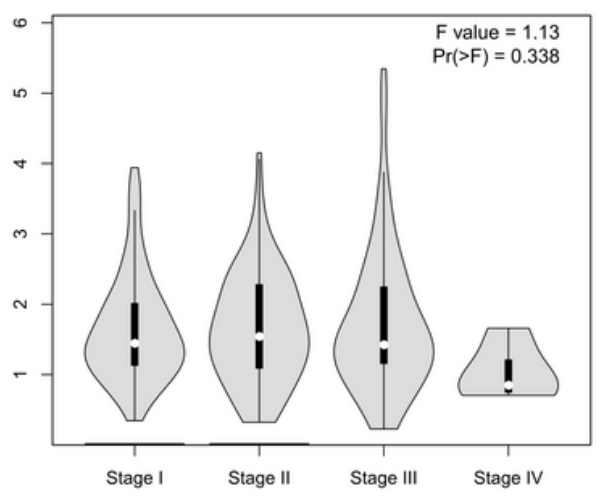

LILRB2

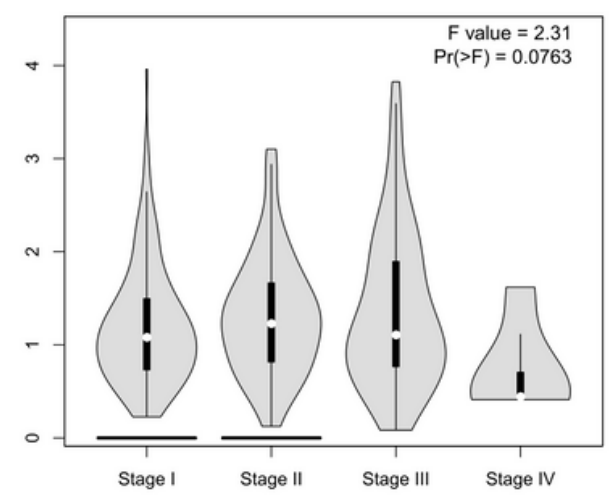

LILRB3

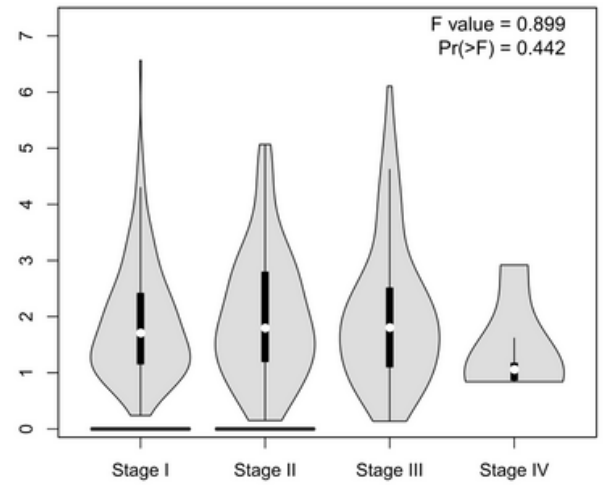

LILRB4

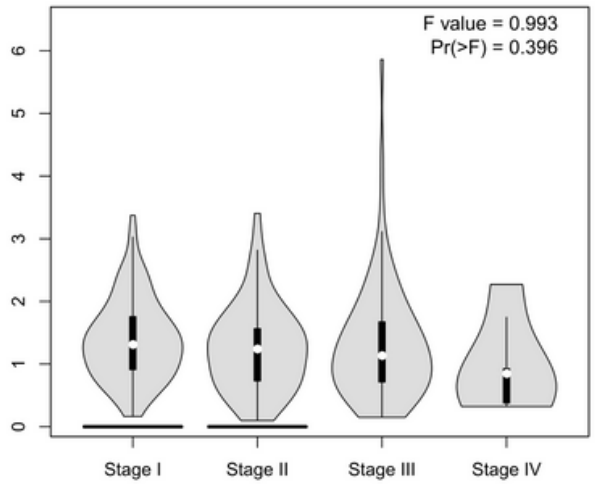

LILRB5

\section{Figure 4}

The correlation between LILRB family expression and tumor stages in liver cancer patients (GEPIA 2.0). The major stages were used for plotting. 
LILRB1

LILRB2

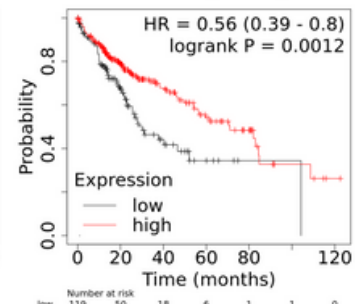

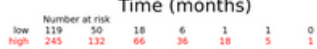

RFS
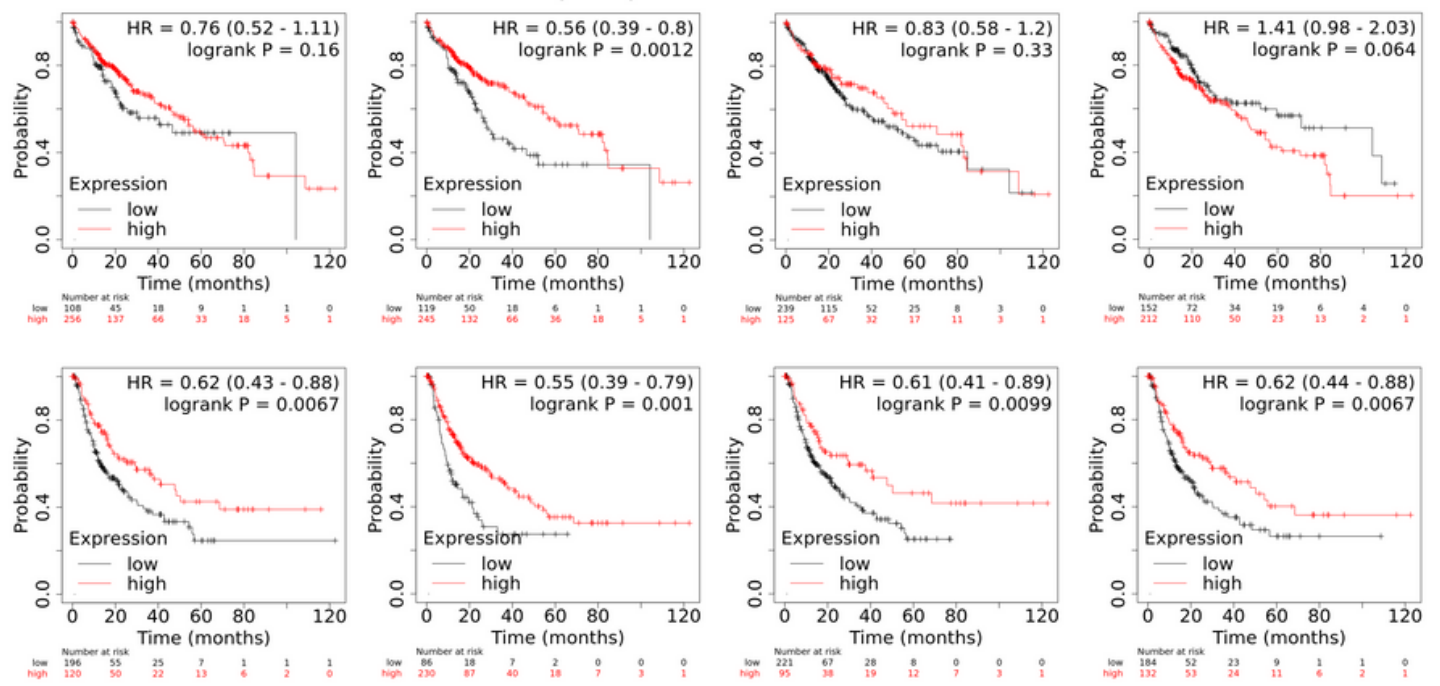

LILRB4

LILRB5

OS
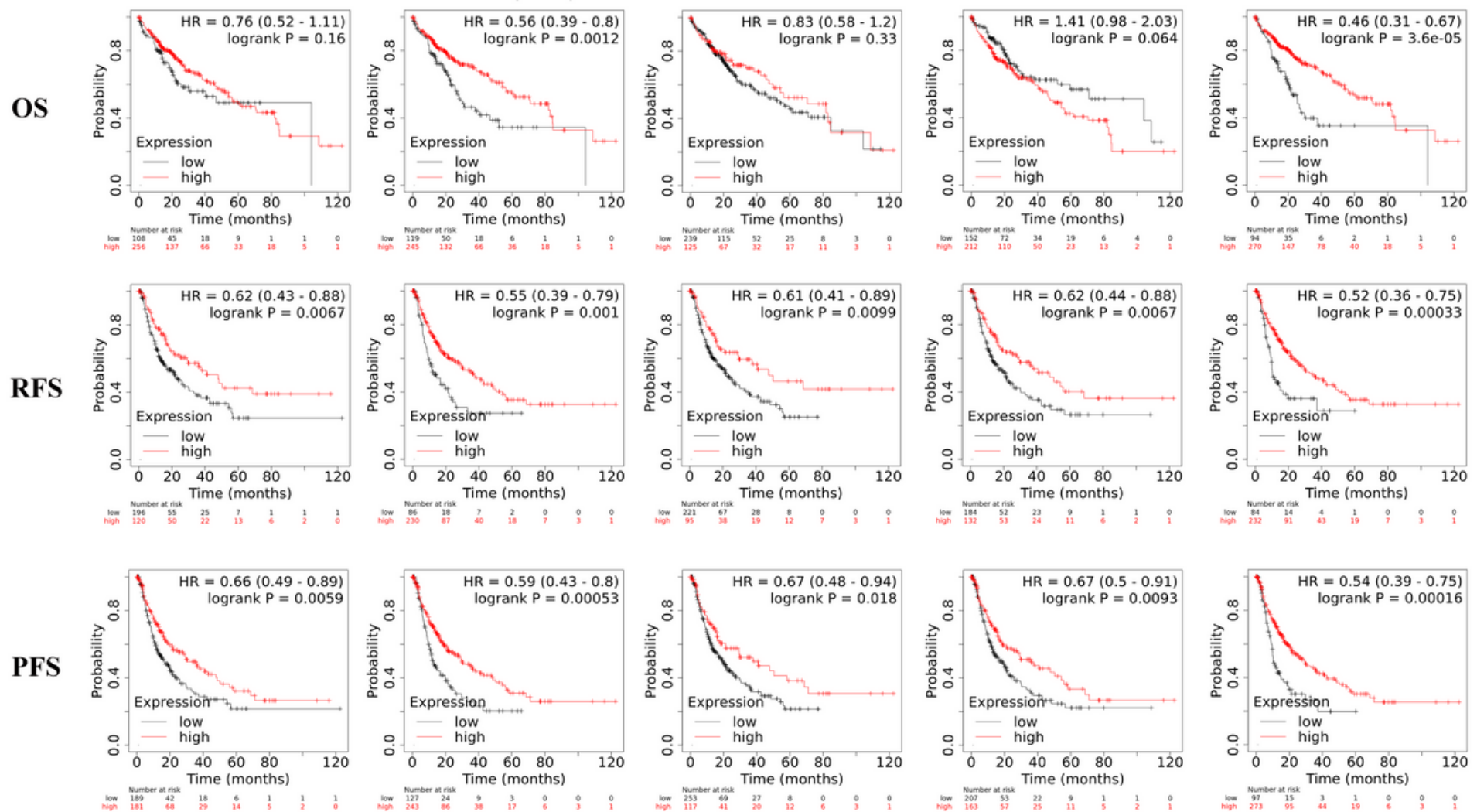

DSS

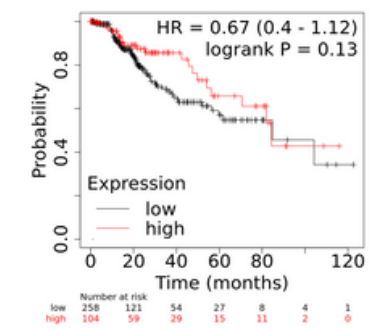

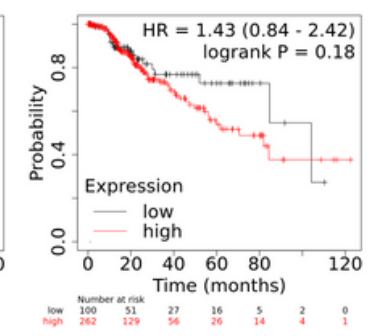

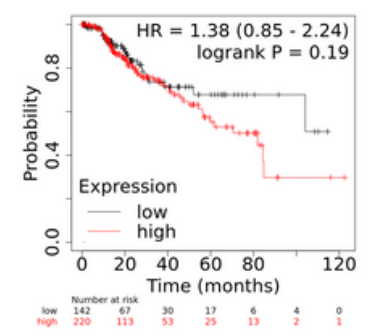

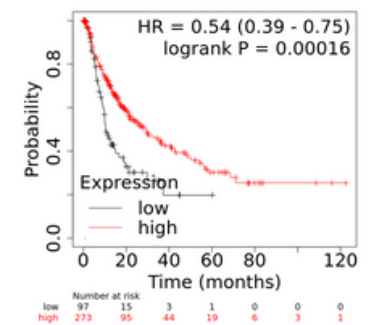

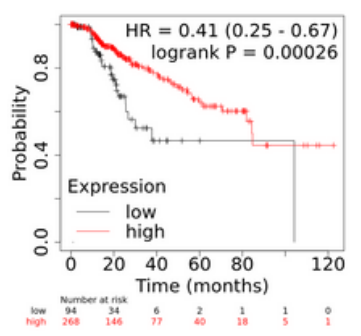

\section{Figure 5}

Survival analyses of the LILRB family in liver cancers (Kaplan-Meier Plotter). The expression of LILRB family members related to OS $(n=364), \operatorname{RFS}(n=316), \operatorname{PFS}(n=370)$ and DSS $(n=362)$ in HCC. Transcriptional expression levels of LILRB family and patient survival information were obtained from GEO, EGA, and TCGA. The best cutoff values were auto-selected by the tool of Kaplan-Meier Plotter. 

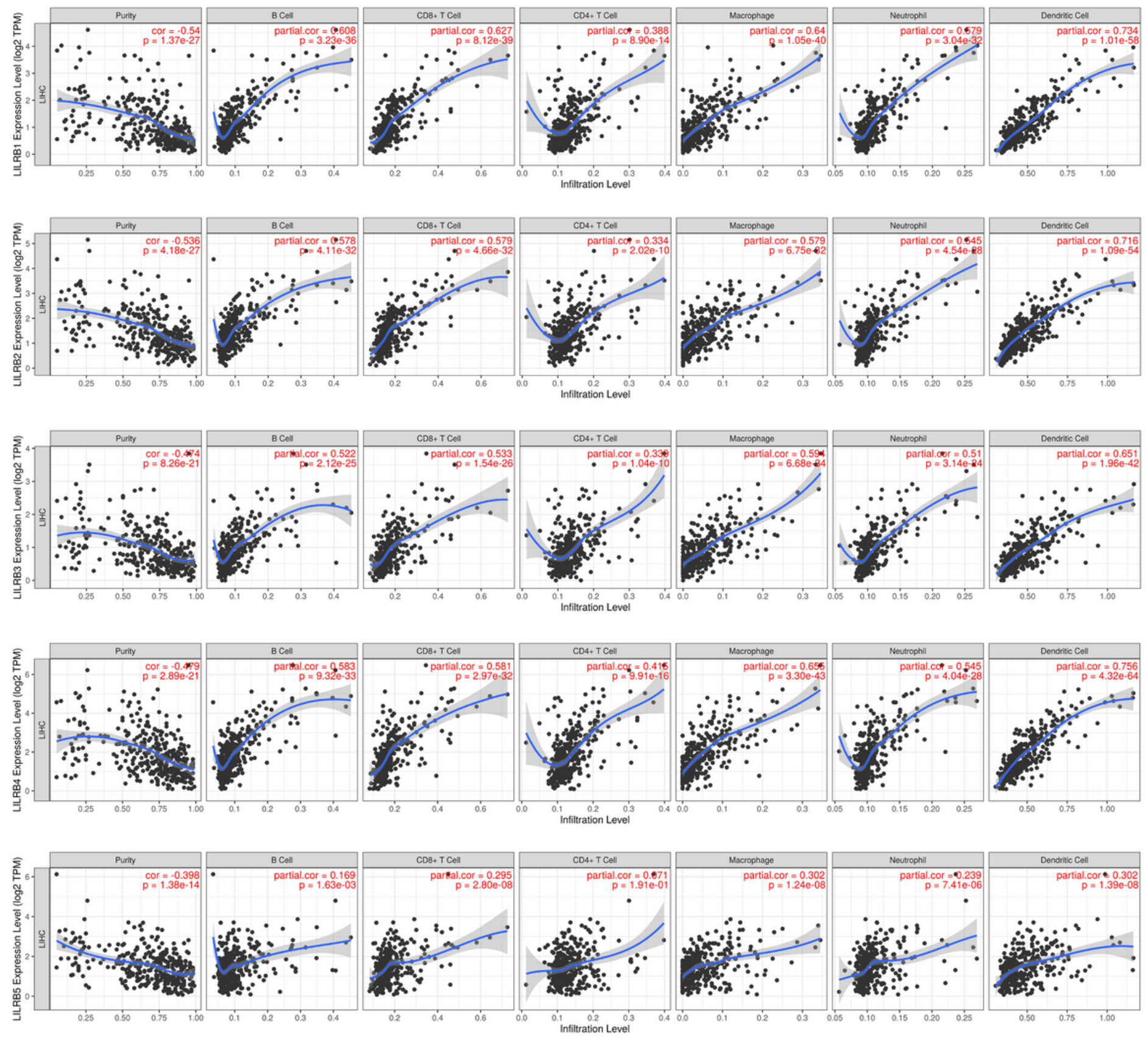

Figure 6

Correlation of LILRB family expression with immune infiltration level in liver cancer (Timer). LILRB family members expression in HCC tissues negatively correlated with tumor purity and positively correlated with infiltration levels of B cells, CD8+ T cells, CD4+ T cells, macrophages, neutrophils, and dendritic cells ( $\mathrm{n}$ $=371)$. 
A

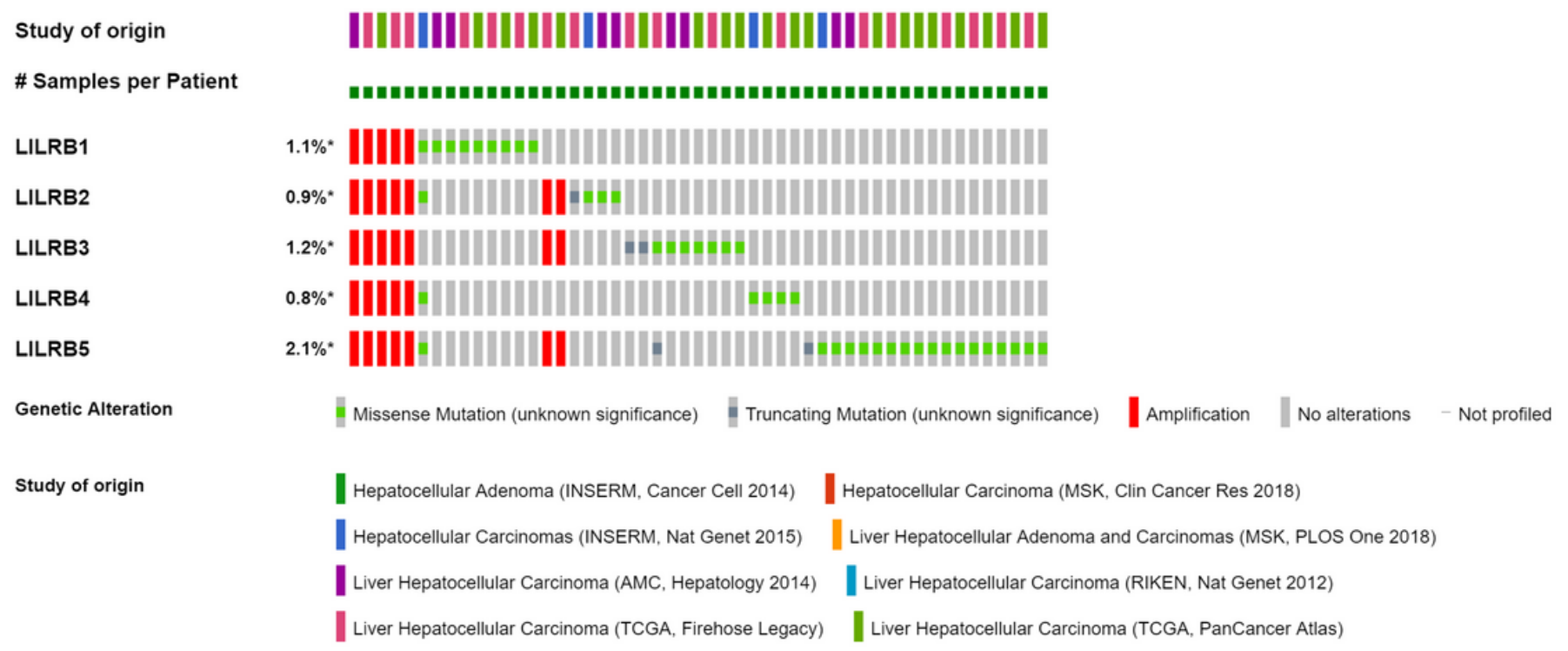

\# Samples per Patient

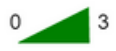

B

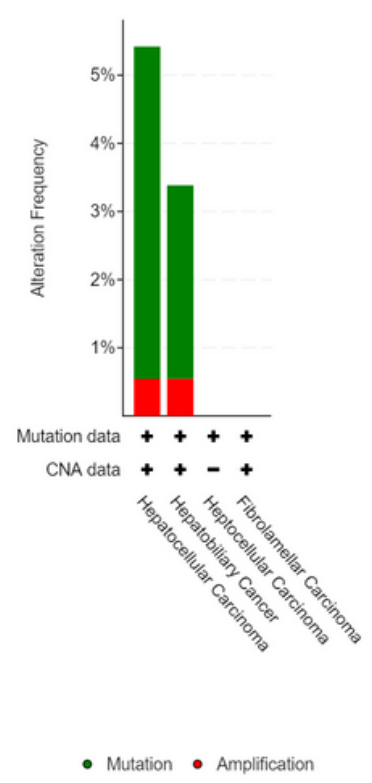

\section{Figure 7}

LILRB family gene expression and mutation analysis in liver cancer (cBioPortal). The data was from 1487 patients and 1507 samples in 8 studies. (A) The mutation types of LILRB family gene. The right part of the figure without alterations was not included. (B) The histogram of the cancer types with mutations in LILRB family genes. CNA means copy number alterations. 


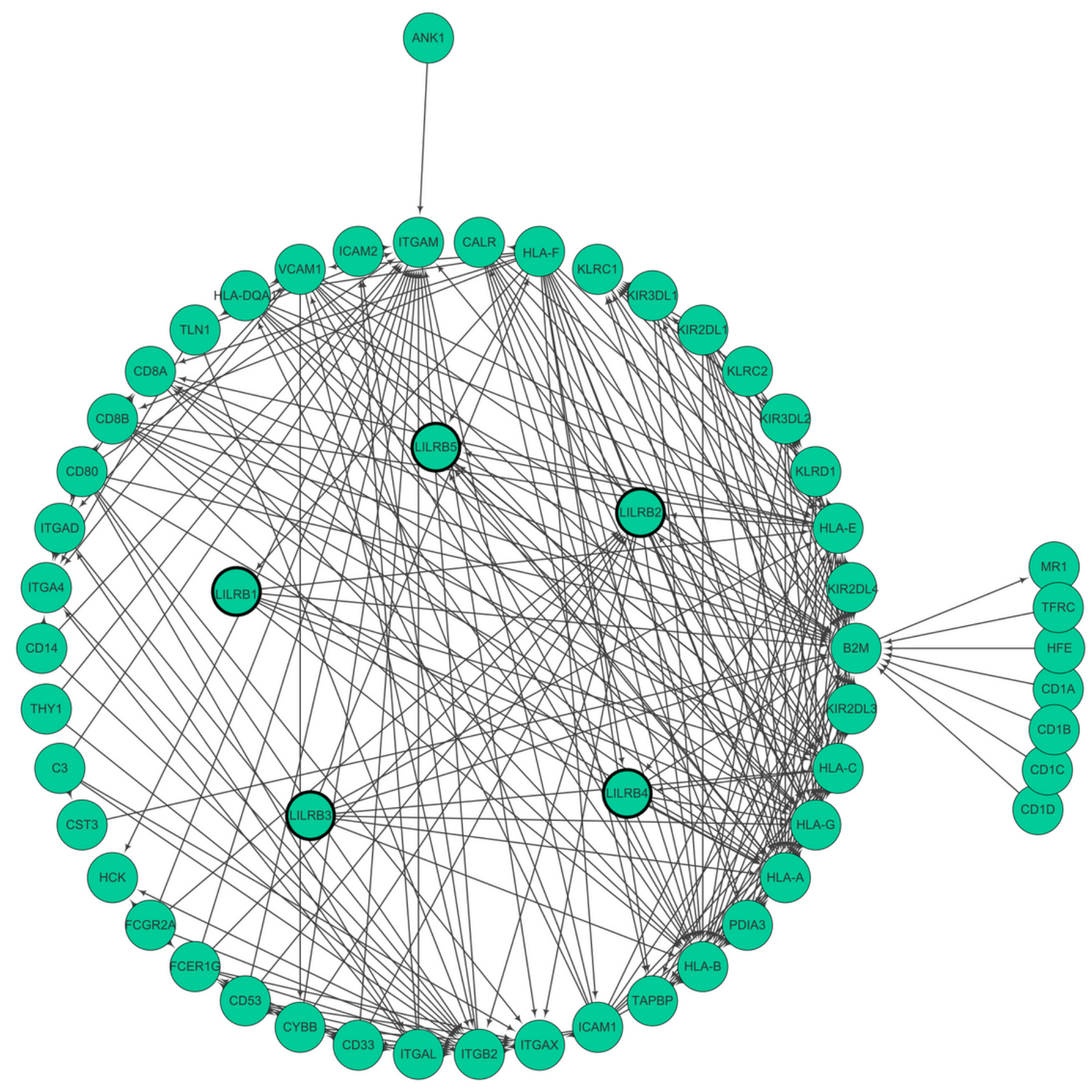

Figure 8

Analysis of LILRB family-related proteins based on a protein-protein interaction network. Using the STRING database, a total of 50 proteins were filtered into the protein PPI network. Nodes represent interacting proteins, and the 452 edges represent known or predicted interactions. 
A

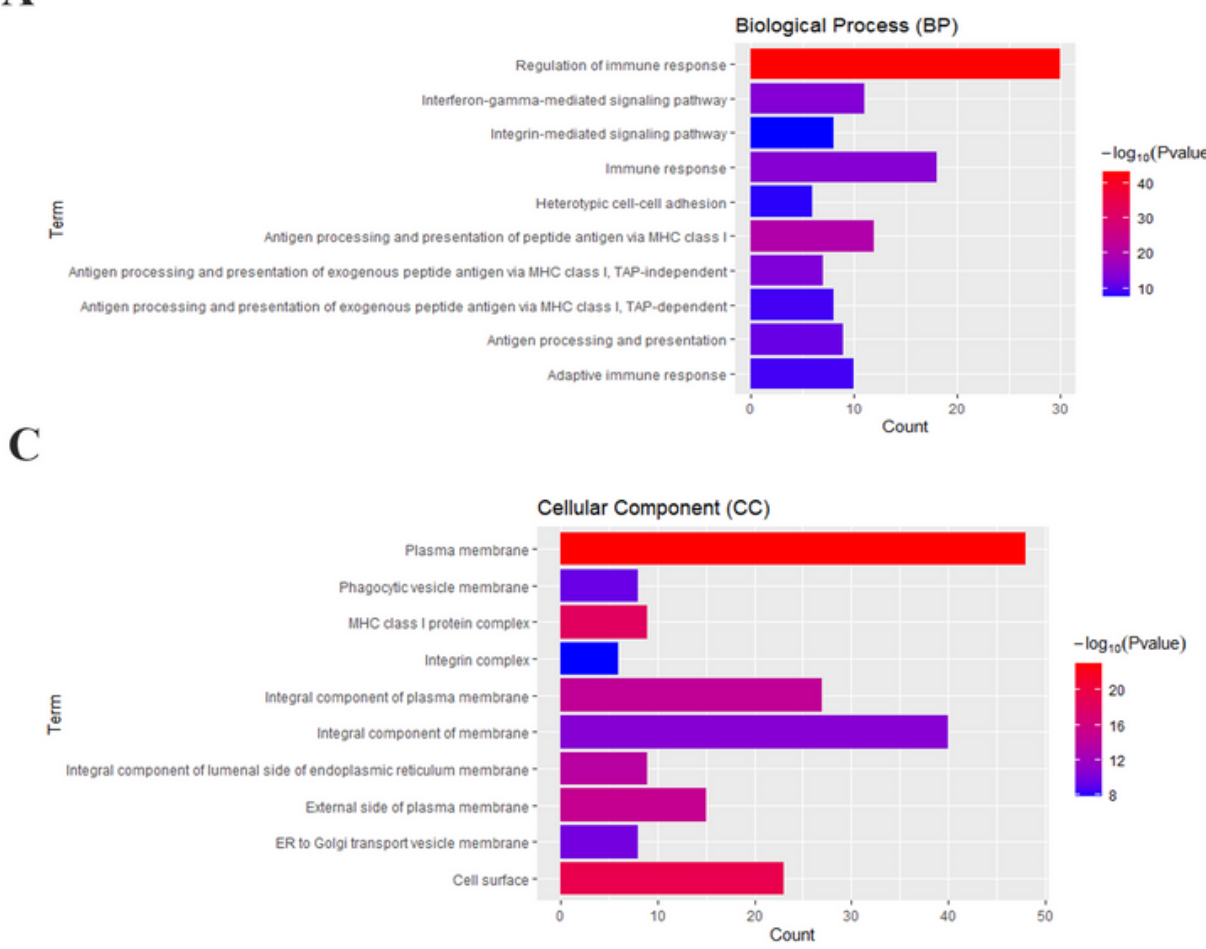

D

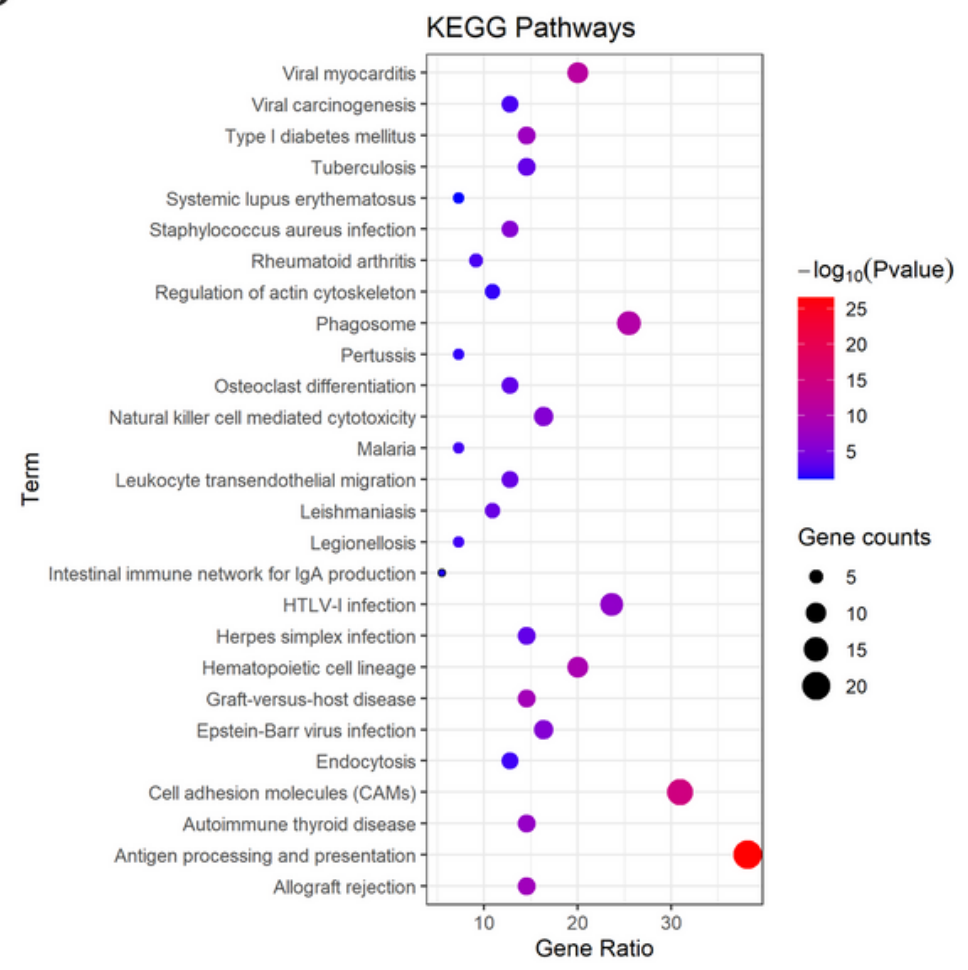

B

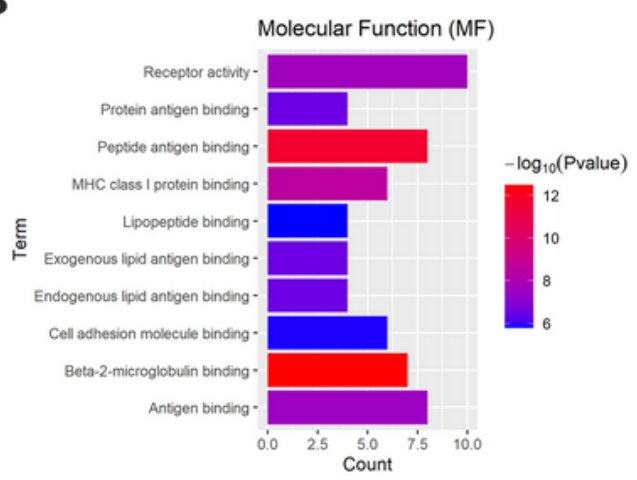

\section{Figure 9}

GO and KEGG analyses of the LILRB family and their related genes. The DAVID online database was used to perform the GO and KEGG analyses. (A), (B) and (C) The top 10 items of the $G 0$ analysis for BP, CC and MF of the LILRB family and their related genes, respectively. (D) The KEGG pathways of the LILRB family and their related genes. 
A

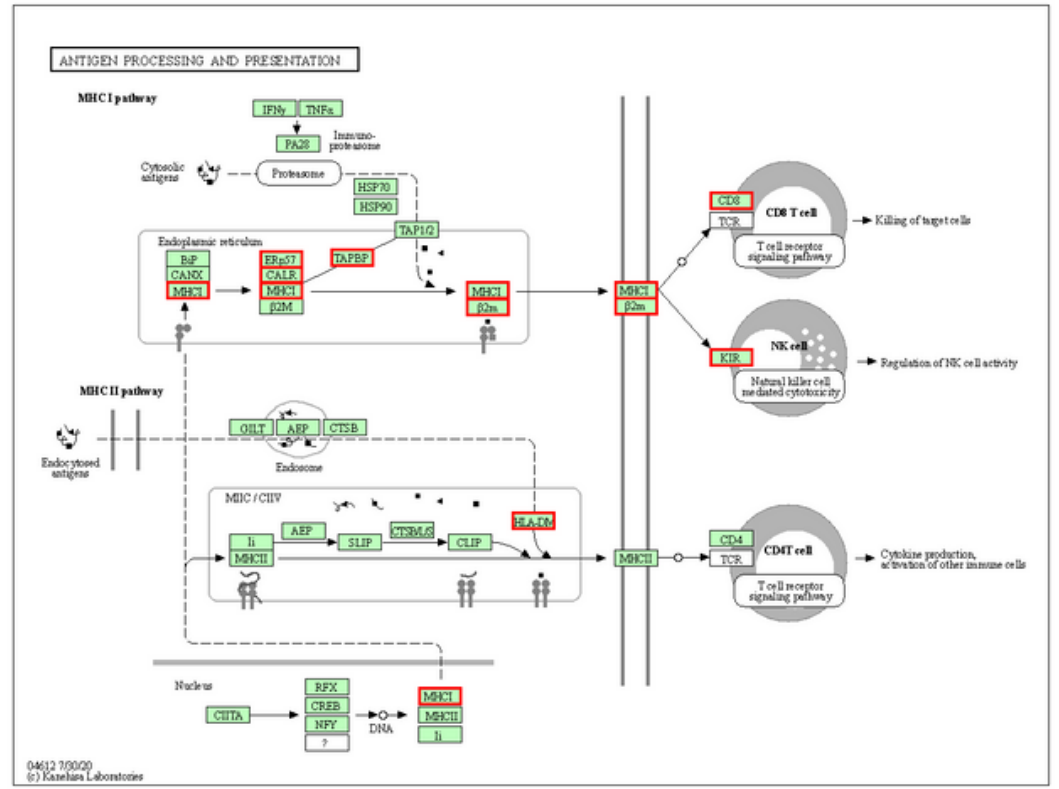

B

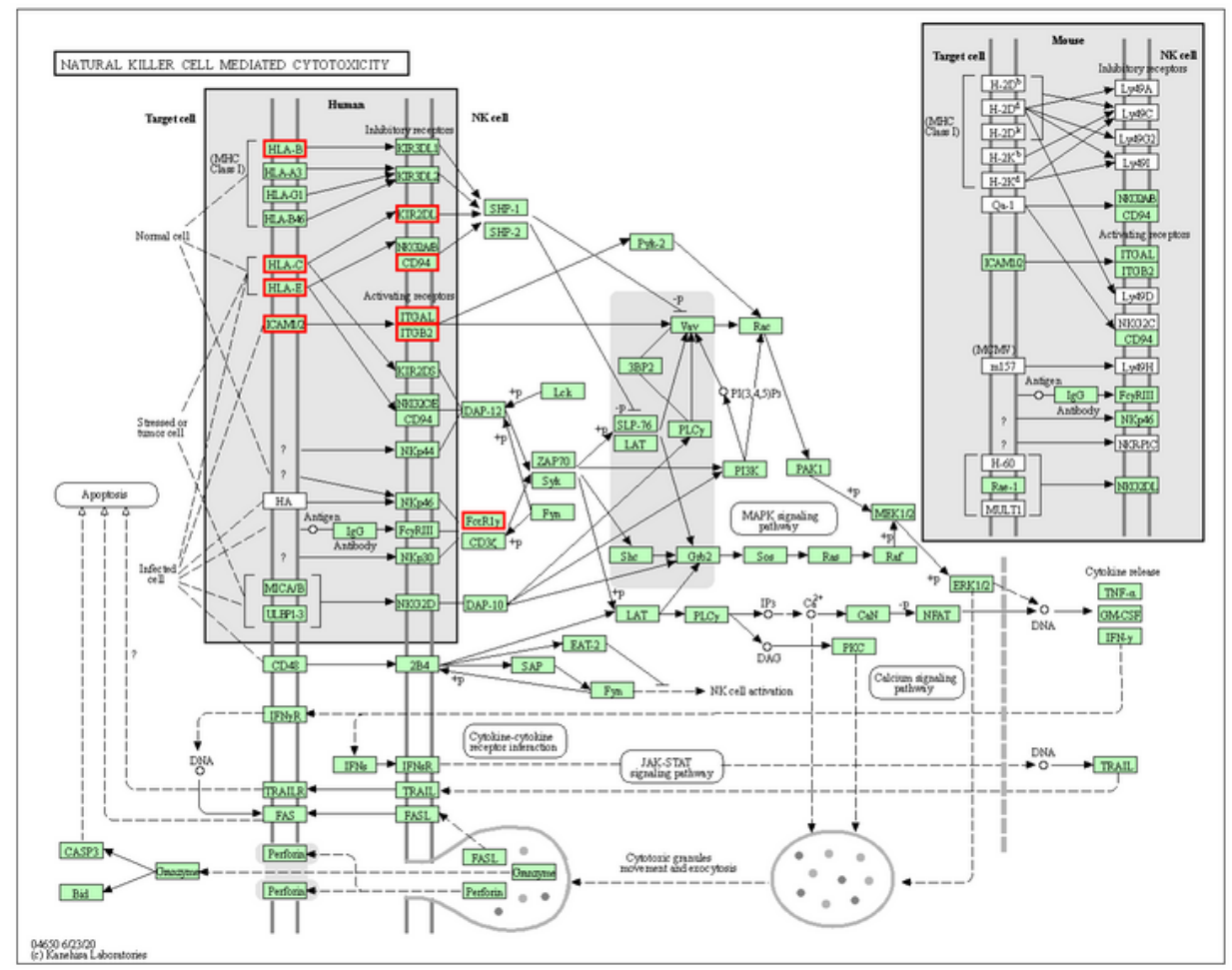

\section{Figure 10}

Antigen processing and presentation and natural killer cell-mediated cytotoxicity pathway of LILRB family-related genes in liver cancer. (A) The antigen processing and presentation signaling pathway; (B) The natural killer cell-mediated cytotoxicity signaling pathway. The red boxes represent LILRB family member-related genes. 
A

Tumor

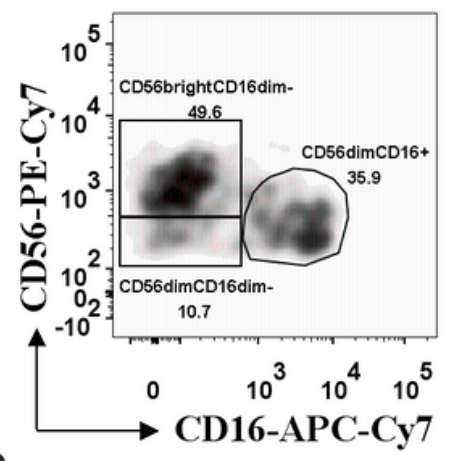

B

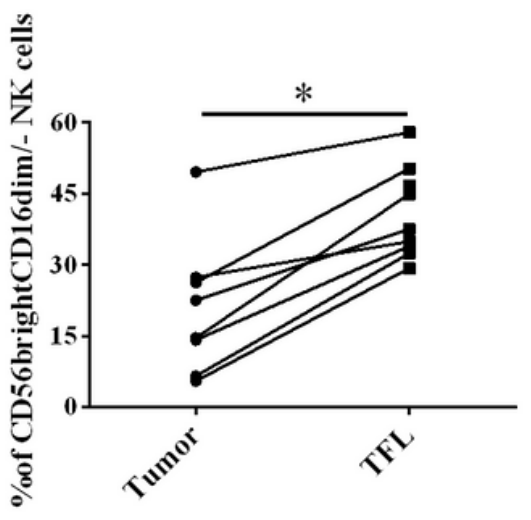

TFL
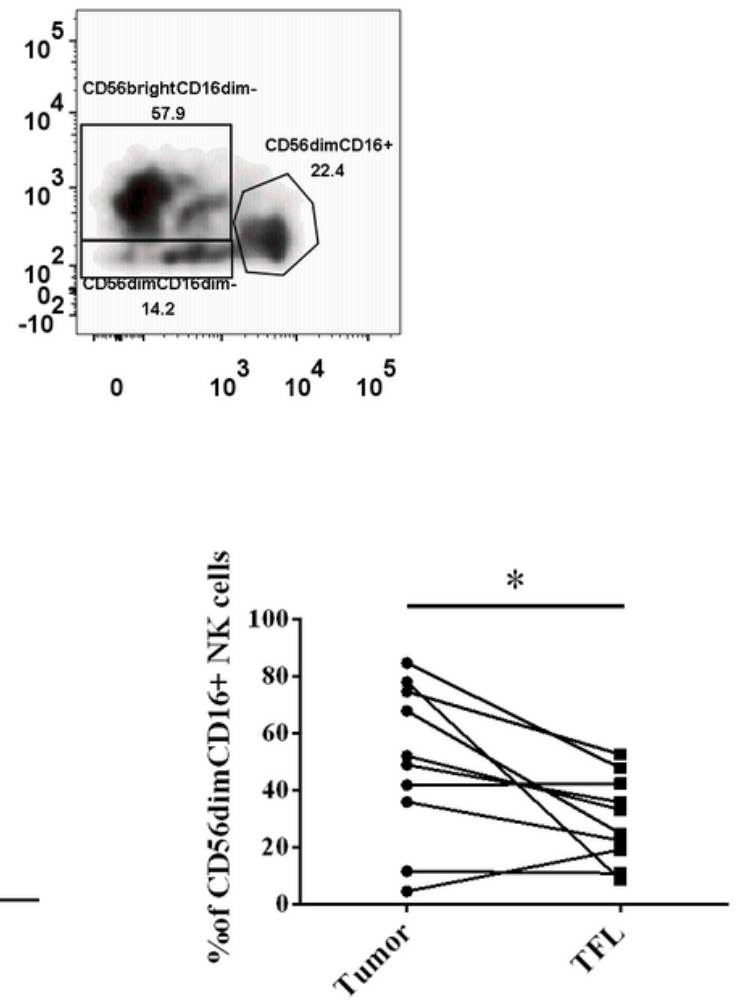

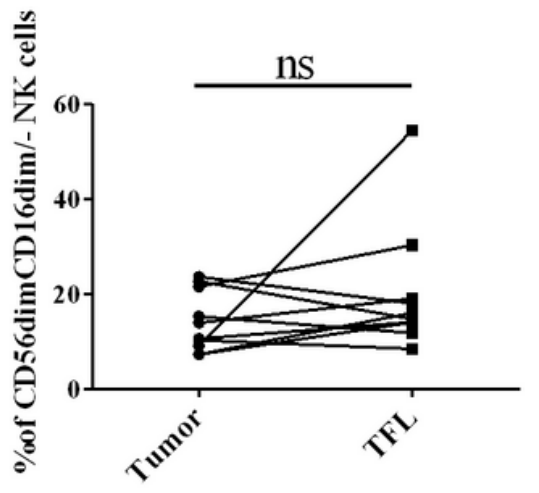

Figure 11

The expression of NK cell subsets in liver cancer. (A) Flow cytometry was used to detect NK cells. NK cell subsets were identified by three unique CD56 and CD16 expression patterns in the gate of Live+CD3CD56bright/dim. (B) The percentage of NK cell subsets from liver tumors and the corresponding TFL $(n=10) .{ }^{*} p<0.05$.

\section{Supplementary Files}

This is a list of supplementary files associated with this preprint. Click to download.

- supplementary.docx 